\title{
Utilisation du numérique à l'école élémentaire : profils d'utilisation et analyse des compétences
}

\author{
Amélie Duguet, Jean-François Giret, Sophie Morlaix
}

amelie.duguet@u-bourgogne.fr jean-francois.giret@u-bourgogne.fr

sophie.morlaix@u-bourgogne.fr

Article publié dans Carrefours de l'éducation, n47, 2019, pp. 175-194.

DOI : 10.3917/cdle.047.0175

Depuis plusieurs années, de nombreux travaux scientifiques s'interrogent sur les effets des technologies de l'information et de la communication pour l'enseignement (TICE). La variété des contextes et des situations pédagogiques conduit souvent à des résultats nuancés voire contradictoires depuis trente ans (Poyet, 2009 ; Thibert, 2012). Leur effet varie également en fonction du type de TICE et du contexte d'intégration de ces dernières (Karsenti et al, 2005). Enfin, les différences en matière de méthodologie rendent peu aisées les comparaisons entre les recherches produites (Karsenti et al., 2005). Dès lors, la question de l'efficacité des TICE devient « redoutable », produisant des «partisans » et des «adversaires » des TICE (PoutsLajus, 2001). Pourtant, les technologies numériques sont investies en France depuis plusieurs années par les pouvoirs politiques comme un instrument de lutte contre l'échec scolaire. L’hypothèse est parfois faite que les TICE améliorent sensiblement la qualité de l'éducation en modifiant et facilitant pour certains les conditions d'apprentissage des élèves. Les compétences liées à l'outil numérique pourraient jouer un rôle central dans ses conditions d'apprentissage et réduire in fine les inégalités. Cependant, cela ne serait le cas que si elles constituaient les bases d'une capacité d'apprendre à apprendre plus générale et plus transférable que les compétences techniques liées strictement au TICE (Brotcorne, Valenduc, 2009). Cette question des compétences générales ou spécifiques susceptibles d’être développées dans un cadre scolaire se pose également dans le cadre extrascolaire où les élèves développent des pratiques numériques plus ou moins intenses.

Ce travail propose d'apporter un éclairage à partir d'une enquête réalisée auprès de 1400 élèves en fin de primaire en France ainsi que d'une cinquantaine d'enseignants dans un département français. L'objectif est d'étudier les facteurs, contextuels notamment, contribuant au développement de différentes compétences qui peuvent être liées à l'outil numérique. La recherche est structurée en quatre parties. La première partie rappelle quelques éléments de la littérature sur les effets du numérique sur les compétences et la réussite des élèves. La deuxième partie présente l'enquête réalisée et quelques éléments de statistiques descriptives concernant l'utilisation des TICE. La troisième partie approfondit cette première analyse en 
construisant une typologie des pratiques numériques des élèves à l'école et en dehors de l'école. Enfin, une quatrième partie cherche à comprendre comme se forment les compétences numériques des élèves, en fonction de leurs caractéristiques mais également en fonction de celles de leurs professeurs et des ressources disponibles dans la salle de classe.

\section{TICE et compétences des élèves}

\section{Les TICE à l'école : des effets sur les élèves qui font débat}

Les résultats de recherche portant sur l'évaluation des TICE ont suscité de nombreux débats qui peuvent parfois conduire à quelques doutes sur leur efficacité. De nombreux travaux, comme le souligne Poyet (2009), ont pourtant mis en avant le rôle joué par les TICE dans l'amélioration de la motivation et de l'attention des élèves. D'autres (Barette, 2004) indiquent que l'utilisation des nouvelles technologies à la maison et en classe conduirait les élèves à obtenir de meilleures performances aux tests nationaux, notamment en mathématiques et en anglais. Néanmoins, des méta-analyses semblent indiquer que les technologies auraient en réalité un impact modéré, voire inexistant et parfois négatif, sur les résultats des élèves (Michko, 2007). D'après un rapport de l'OCDE en 2015, la technologie constituerait pourtant le seul moyen d'élargir au maximum l'accès à la connaissance. Toutefois, la recension de 355 rapports de recherche par Russel (2001) portant sur l'efficacité des TICE tend à valider la thèse du phénomène "no significant difference ». Autrement dit, il n'existerait pas de véritable preuve d'une meilleure efficacité des apprentissages grâce aux technologies (Chaptal, 2008). D’ailleurs, l'OCDE (2015) considère que les nouvelles technologies ne seraient pas d'un grand secours pour combler les écarts de compétences entre élèves favorisés et défavorisés, les ressources investies dans les TIC dans le domaine de l'éducation n'entretenant même aucun lien avec une amélioration des résultats des élèves en compréhension de l'écrit, en mathématiques et en sciences. Garantir pour chaque enfant l'acquisition d'un niveau de compétence de base en compréhension de l'écrit et mathématiques serait bien plus profitable pour maintenir l'égalité des chances que d'élargir l'accès aux technologies numériques (OCDE, 2015). Toutefois, des auteurs remettent en cause la pertinence de l'analyse de l'impact du numérique sur les résultats : les conditions méthodologiques nécessaires à une évaluation rigoureuse de l'effet de l'introduction d'un outil technologique paraissent délicates à réunir (Barette, 2004). Il serait par conséquent préférable d'étudier les conditions pédagogiques dans lesquelles ces usages ont lieu (Thibert, 2012). En effet, d'après le rapport de la mission Fourgous (2010), la technologie pourrait être utilisée au service des nouvelles pédagogies en rendant les apprenants davantage acteurs de leurs apprentissages et en favorisant la mise en œuvre de pédagogies différenciées. La maîtrise technique et pédagogique des TICE par les enseignants constituerait en ce sens un facteur clé dans la compréhension de la réussite des élèves. Néanmoins, pour l’OCDE (2015), certains enseignants ne maîtriseraient pas encore suffisamment le type d'approches pédagogiques permettant de tirer pleinement profit des nouvelles technologies. De plus, si l'équipement en TICE peut paraître important au sein des établissements scolaires, les usages 
pédagogiques des technologies en classe sont souvent plus limités. La DEPP (2010) ${ }^{1}$ indique que 95\% des enseignants utilisent les TICE à des fins professionnelles mais seulement 19\% les mobilisent en présence des élèves et $11 \%$ permettent à leurs élèves de les utiliser euxmêmes. Ce faible usage pédagogique des TICE par les enseignants serait à mettre en lien avec la multiplicité des innovations pédagogiques qui ne laisserait pas suffisamment de temps aux enseignants pour s’y adapter (Depover, 2010), ainsi qu'à la formation des enseignants souvent considérée comme trop «techno-centrée ». Celle-ci représenterait alors un levier essentiel pour que le numérique soit mobilisé de manière efficace (Thibert, 2012). On peut néanmoins s'interroger sur l'ensemble des savoirs susceptibles d'être produits lors des usages numériques, qu'ils soient d'ailleurs acquis dans un cadre scolaire ou extra-scolaire. Quels sont ceux parmi eux, qui pourraient se révéler suffisamment transférables pour garantir une certaine efficacité dans les conditions d'apprentissage ?

\section{Quelles compétences numériques des élèves?}

Les générations actuelles d'élèves sont régulièrement qualifiées de "digital natives » (Prensky, 2001), en raison du fait qu'elles sont nées dans un environnement fortement numérique, au contraire de leurs aînés qui ont dû s’adapter au numérique. Ainsi, pour ces générations, la culture numérique fait partie intégrante de la culture juvénile. Ces écoliers seraient alors plus enclins à apprendre avec les technologies, «car ces dernières seraient constitutives de leur mode de vie » (Collin, Karsenti, 2012). Pourtant, des auteurs indiquent qu'une telle croyance est en réalité infondée (Roland, Choumane, Vanmeerhaeghe, 2016), en raison de l’hétérogénéité de cette génération en termes de compétences numériques, ces dernières étant étroitement liées au capital social, scolaire et culturel de l'élève (Granjon, 2003). De ce fait, si une partie des jeunes a tendance à surévaluer leurs compétences numériques par rapport à leurs aînés (Dauphin, 2012), les compétences développées par les élèves dans leurs pratiques personnelles se distingueraient en réalité par leur caractère « limité et local » (Fluckiger, 2008). Leur grande habileté sur certains logiciels utilisés au quotidien cacherait de véritables lacunes, leurs compétences n'étant pas transférables sur d'autres logiciels. Ces constats rejoignent ceux de Baron et Bruillard (2008), qui à l'appuie d'une recension des travaux produits sur le sujet indiquent que l'utilisation des technologies par les jeunes est fréquente mais reste «dans un spectre très limité et avec un degré d'autonomie relatif »(p.9).

Les compétences techniques, les savoir-faire techniques et conceptuels manqueraient souvent aux élèves (Dauphin, 2012). En outre, la culture numérique des élèves se construit le plus souvent en dehors de l'école (Fluckiger, 2008). En résulte un profond décalage entre les usages prescrits par l'école et les usages personnels, Dauphin (2012) allant même jusqu’à employer le terme de «contradiction » entre ces deux types d'usage. Ce dernier constate que les élèves ont principalement un rapport consumériste aux TICE et des usages ludiques peu transférables au domaine scolaire, notamment en raison d'une temporalité différente dans les usages : des usages personnels importants s’opposent à des usages scolaires contraints par des

\footnotetext{
${ }^{1}$ Cité par Thibert (2012).
} 
délais temporels et de planification de l'activité d'apprentissage (Fluckiger, 2008). D’ailleurs, la culture numérique juvénile se caractérise, "de façon caricaturale ", à "savoir utiliser Facebook et MSN, savoir se présenter sur un chat et naviguer sur le web de manière ludique » (Dauphin, 2012). Par conséquent, les usages extrascolaires sont jugés comme insuffisants en termes de maîtrise technique dans la sphère de l'école (Dauphin, 2012), ce décalage pouvant toutefois en partie être imputé à une différence dans les outils utilisés en classe et à la maison. Le ministère de l'éducation nationale avait d'ailleurs préconisé en 2007 que soit proposé un accompagnement éducatif en matière de TIC en dehors du temps scolaire.

\section{Vers une approche empirique des facteurs explicatifs des compétences numériques des élèves}

\section{Problématique}

Malgré ces débats, peu de travaux, à notre connaissance, se sont attachés à examiner, de manière empirique, à la fois le rôle joué par des facteurs personnels et des facteurs contextuels dans les compétences numériques développées par les élèves à l'école primaire. C'est l'objectif assigné à la présente recherche. Par compétences, entendons l'ensemble des «aptitudes, connaissances générales et professionnelles, théoriques et pratiques, de nature plutôt intellectuelle, savoirs, savoir-faire, savoir être qui conditionnent un comportement, notamment la réussite dans une tâche précise, et qui se vérifient dans une épreuve concrète » (Dadoy, 1999). Si l'on s'intéresse à la signification de ce concept davantage dans le cadre de l'usage des TIC, Vandeput et Henry (2012) indiquent que la compétence revient à mobiliser « des savoirs et des savoir-faire à des fins de résolution d'un problème ou d'obtention d'un résultat comme, par exemple, un produit numérique d'une qualité préalablement exigée » (p.4). La recommandation du parlement européen et du conseil sur les compétences clés pour l'éducation et la formation tout au long de la vie, datant du 18 décembre 2006, permet de préciser la définition du concept de compétence numérique : celle-ci impliquerait ainsi un « usage sûr et critique des technologies de la société de l'information (TSI) au travail, dans les loisirs et dans la communication » et nécessiterait comme condition préalable la maîtrise des TIC, à savoir « l'utilisation de l'ordinateur pour obtenir, évaluer, stocker, produire, présenter et échanger des informations, et pour communiquer et participer via l'internet à des réseaux de collaboration ${ }^{2}$. De manière plus brève, Brotcorne et Valenduc (2009) définissent les compétences numériques comme faisant référence à « la capacité à utiliser les TIC de manière efficace et autonome » (p.52). Or, ces compétences numériques peuvent être de plusieurs types. Ainsi, le «décret mission » voté le 24 juillet 1997 en communauté française de Belgique et depuis régulièrement mis à jour, distingue les compétences disciplinaires (à acquérir dans une discipline scolaire), des compétences terminales (leur maîtrise à un niveau donné est attendue à la fin de l'enseignement secondaire) et des compétences transversales ( "attitudes, démarches mentales et démarches méthodologiques communes aux différentes disciplines »). De même, Frinking et al. (2005) ont distingué trois types de compétences numériques, repris par Baron et Bruillard (2008) : les compétences « nécessaires pour utiliser efficacement les outils informatisés présents dans l'entreprise », "les compétences de praticien en TIC » (pour installer, maintenir...) et « les compétences métier, nécessaires pour

\footnotetext{
${ }^{2}$ http://eur-lex.europa.eu/legal-content/FR/TXT/?uri=celex:32006H0962
} 
exploiter les potentialités des technologies » (p.8). Pour notre part, nous faisons davantage le choix de nous appuyer sur les travaux de Brotcorne et Valenduc (2009) qui mentionnent l'existence de trois catégories de compétences numériques: les compétences « instrumentales » se réfèrent à la manipulation du matériel et des logiciels par l'individu. Les compétences «structurelles ou informationnelles » ont trait à la façon «d'entrer dans les contenus en ligne », ou autrement dit de " chercher, sélectionner, comprendre, évaluer, traiter l'information » (p.53). Les compétences «stratégiques» se rapportent quant à elles à " l'aptitude à utiliser l'information de manière proactive » (p.54). Ces propos laissent à penser qu'il existerait donc des compétences numériques techniques et des compétences plus conceptuelles. Par conséquent, nous considérons pour notre part :

- les compétences techniques, qui relèvent plutôt de la connaissance et de la maîtrise du matériel informatique,

- les compétences non techniques, parmi lesquelles nous différencions la conceptualisation d'internet (représentation que l'élève se fait d'internet) et les compétences éthiques (connaissance des droits et devoirs sur internet).

L'objet de cette recherche est d'analyser la nature de ces compétences au regard des caractéristiques personnelles des élèves, de leurs usages du numérique à la maison et des caractéristiques des enseignants. Plus précisément, ce travail se propose d'apporter des éléments de réponse aux questions suivantes :

- Quelles sont les pratiques numériques des élèves en dehors de l'école ?

- Quels sont les usages du numérique à l’école déclarés par les enseignants ?

- Quels sont les facteurs explicatifs des compétences numériques, techniques et non techniques, développées par les élèves ?

En d'autres termes, notre objectif est de produire une description récente et exhaustive des technologies mobilisées par les élèves en dehors de l'école et des usages qu'ils en font. Si des travaux sur le sujet existent déjà (Fluckiger, 2008 ; Dauphin, 2012), ceux-ci existent depuis plus de cinq années. Il nous paraît donc opportun de renouveler ce type de recherche, tant les technologies sont en perpétuelle évolution. Il en est de même concernant les usages du numérique par les enseignants : si des chercheurs ont déjà travaillé sur la question concernant les enseignants du secondaire (DEPP, 2010 ; Bernet, Karsenti, 2013), rares sont ceux traitant de la situation des enseignants du primaire dans l'hexagone, alors même que les incitations politiques à mobiliser les TICE pour enseigner et l'engouement actuel pour les tableaux blancs interactifs) et les tablettes conduisent à s'interroger sur les équipements des écoles en la matière et les ressources numériques dont les enseignants font usage pour enseigner. Enfin, la question de l'analyse des facteurs, à la fois intrinsèques aux élèves et contextuels, influant sur les compétences numériques de ces derniers s'avère cruciale pour plusieurs raisons : on peut premièrement se demander si la mise en place du nouveau socle commun de connaissances, de compétences et de culture en 2015, faisant la part belle aux technologies ${ }^{3}$, a

\footnotetext{
${ }^{3}$ Le domaine 2 du socle porte sur « les méthodes et outils pour apprendre ». Il est indiqué que l'élève doit savoir « utiliser de façon réfléchie des outils de recherche, notamment sur Internet », qu’il doit apprendre à « utiliser avec discernement les outils numériques de communication et d'information qu'il côtoie au quotidien », à développer une « culture numérique », à identifier « les différents médias (presse écrite, audiovisuelle et Web) »,
} 
déjà pu influer sur les compétences numériques des élèves et rendre celles-ci moins superficielles. De plus, la mise au jour des facteurs influant sur ces compétences permettra de fournir des pistes de réflexion concernant les solutions à mettre en œuvre pour améliorer ces dernières. En outre, l'efficacité des TICE sur les apprentissages des élèves reste rarement étudiée en France, et le plus souvent par le prisme de la psychologie du développement (Baron, Bruillard, 2008). Aussi notre travail permettra-t-il d'apporter de nouveaux éléments de compréhension concernant les mécanismes à l'œuvre dans le rôle que peuvent jouer les technologies sur les acquisitions des élèves, ici en termes de compétences numériques.

\section{Les outils d'enquête}

Afin d'apporter des éléments de réponse à ces différentes questions, une enquête par questionnaires a été menée dans 37 écoles élémentaires de Côte d’Or, auprès de l'ensemble des élèves de CM1 et de CM2 et de leurs enseignants. L’échantillon d'écoles, conçu en coopération avec les services du DASEN (Directeur académique des services de l'éducation nationale), est représentatif des écoles du département. Les passations sous forme papier se sont déroulées en classe entière au cours de l'année 2016. Le questionnaire adressé aux élèves comportait des dimensions liées à leurs caractéristiques sociodémographiques, leur équipement et leurs usages du numérique à la maison. De plus, des items ont permis de mesure leur niveau de compétences numériques, techniques et non techniques ${ }^{4}$. Afin de limiter le taux de non réponse et les réponses fantaisistes de la part des élèves en raison d'une lassitude, ces compétences ont été mesurées à partir d'un faible nombre de questions (8 questions pour les compétences techniques, 7 questions pour les compétences non techniques). Le questionnaire destiné aux enseignants avait pour but de recueillir des données concernant leurs caractéristiques personnelles (genre, âge, ancienneté, formation, niveau de compétences en TICE), les caractéristiques générales de la classe (niveau, nombre d'élèves...), le matériel pédagogique mis à leur disposition dans l'école, leur connaissance et leur utilisation des TICE dans l'apprentissage.

\section{Caractéristiques des élèves et des enseignants de l'échantillon}

L'échantillon d'élèves se compose de 1401 individus de CM1 et de CM2 avec une légère surreprésentation des CM2 (56,7\%). La plupart des élèves (97,9\%) sont âgés de 9 à 11 ans. Par ailleurs, 49 enseignants ont complété le questionnaire qui leur était adressé. Leurs réponses ont ensuite pu être appariées avec la base élève pour environ $60 \%$ de l'échantillon. Un peu plus de 65\% des enseignants déclarent avoir été formés aux TICE. Cette formation est très récente (2015 ou 2016) pour 15\% des individus.

\footnotetext{
à mobiliser « différents outils numériques pour créer des documents intégrant divers médias et les publier ou les transmettre » ou bien encore à « communiquer notamment par le biais des réseaux sociaux dans le respect de soi et des autres ».

${ }^{4}$ Nous décrivons ultérieurement les réponses apportées par les élèves concernant leurs compétences numériques, permettant ainsi au lecteur de connaître la nature des items proposés.
} 


\section{Les pratiques numériques des élèves en dehors de l'école}

\section{État des lieux descriptif}

Les réponses apportées par les élèves au questionnaire ont permis d'appréhender la nature du matériel dont ils disposent et de leurs pratiques numériques en dehors du cadre scolaire. Ainsi, la télévision, l'ordinateur et le téléphone portable représentent des outils possédés par plus de $90 \%$ des élèves, la tablette et la console vidéo par environ $86 \%$. En ce qui concerne plus précisément l'utilisation de l'ordinateur par les élèves, 60,2\% doivent demander l'autorisation avant de l'utiliser, même si l'adulte est assez rarement présent à côté d'eux lorsqu'ils utilisent l'ordinateur. Ce sont par ailleurs le plus souvent les parents qui ont appris aux enfants à utiliser l'ordinateur (48,6\%), bien qu'une proportion non négligeable (20,6\%) déclare avoir appris seule. Les motifs d'utilisation de l'ordinateur sont prioritairement le visionnage de vidéos cité par 78,2\% des élèves, l'écoute de musique $(69,7 \%)$ et le fait de jouer à des jeux vidéos (66\%). Le fait de se rendre sur les réseaux sociaux ne figure pas parmi les raisons les plus évoquées. Cette situation peut sans doute s'expliquer par le fait que près d'un tiers des élèves (31\%) ne sont inscrits sur aucun réseau social. A contrario, 48,6\% le sont sur youtube, $32,8 \%$ sur skype, $25,3 \%$ sur snapchat et $22,8 \%$ sur facebook. La majorité des élèves déclare avoir l'accord de leurs parents pour l’inscription sur un réseau social.

\section{Typologie des pratiques numériques des élèves}

Afin d'identifier des profils d'utilisateurs du matériel numérique à la maison, nous avons construit une typologie statistique à partir de la méthode proposée par Isnard et Sautory (1994). L'objectif a été dans un premier temps de mener une analyse en composante multiple $(\mathrm{ACM})$ à partir des différentes variables concernant les pratiques du numérique des élèves chez eux ${ }^{5}$. Nous nous sommes focalisés sur les pratiques accordant un rôle central à l'ordinateur, même si d'autres questions ont été introduites. L'intérêt a été de repérer à la fois les motifs d'utilisation mais également, les durées d'utilisation ainsi que l'implication éventuelle d'adultes pour l'aide ou la surveillance.

Les 10 premiers axes factoriels, issus de l'ACM, représentent 53\% de l'inertie cumulée mais seulement trois axes ont une inertie de $5 \%$ ou plus. Le premier axe qui regroupe $16 \%$ de l'inertie, concerne l'utilisation fréquente ou non des réseaux sociaux. Il oppose des jeunes qui n'utilisent jamais les réseaux sociaux et les utilisateurs de Facebook ou d'Instagram, de Skype et de Youtube. Le deuxième axe, qui correspond à 5\% de l'inertie, permet de distinguer certaines pratiques d'utilisation des ordinateurs. Ces pratiques concernent notamment l'utilisation de logiciel éducatif, l'enregistrement et la transformation de photos mais aussi le recours à l'ordinateur pour la préparation de devoirs et pour la lecture. Elles s'opposent à d'autres pratiques, comme par exemple l'utilisation de l'ordinateur pour regarder des vidéos. Le troisième axe, qui est égal à un peu moins de $5 \%$ de l'inertie, capte la fréquence d'utilisation d'internet et oppose les jeunes qui ont une utilisation fréquence de l'ordinateur durant la journée et les vacances scolaires à ceux qui en ont une utilisation plus limitée. Pour ces derniers, l'autorisation d'un adulte avant l'utilisation semble obligatoire.

\footnotetext{
5 De manière générale, les utilisations de console vidéo ou d’un téléphone portable semblent moins discriminantes dans les premiers axes factoriels et affectent peu les résultats de la CAH lorsqu'ils sont introduits.
} 
Dans un second temps, à partir des axes de l'analyse factorielle, une classification ascendante hiérarchique a été réalisée pour identifier des profils différents d'utilisation du numérique. Seuls les 10 premiers axes ont été retenus. Le dendrogramme (en annexe 2) a conduit à privilégier une partition en 3 classes :

\section{Tableau 1. Classes issues de la typologie}

\begin{tabular}{|c|c|c|l|}
\cline { 2 - 4 } \multicolumn{1}{c|}{} & $\begin{array}{c}\text { Nombre } \\
\text { d'élèves }\end{array}$ & Pourcentage & \multicolumn{1}{c|}{ Variables surreprésentées } \\
\hline $\begin{array}{c}\text { Classe } \\
1\end{array}$ & 708 & $50,5 \%$ & $\begin{array}{l}\text { - Utilisation fréquente d'internet, de Youtube, } \\
\text { Skype } \\
\text { - Autorisation de l'adulte }\end{array}$ \\
\hline $\begin{array}{c}\text { Classe } \\
2\end{array}$ & 465 & $33,2 \%$ & $\begin{array}{l}\text { - Présence et l'autorisation d'un adulte } \\
\text { - Utilisation de l'ordinateur pour les jeux } \\
\text { éducatifs, le travail sur des devoirs, la lecture }\end{array}$ \\
\hline $\begin{array}{c}\text { Classe } \\
3\end{array}$ & 228 & $16,3 \%$ & $\begin{array}{l}\text { - Utilisation des réseaux sociaux : Twitter, } \\
\text { Instagram, Snapchat, MSN ou Facebook } \\
\text { - Fréquence élevée durant les vacances } \\
\text { - Peu/pas de contrôle de l'adulte }\end{array}$ \\
\hline
\end{tabular}

La première classe correspond à une forte utilisation d'internet mais sous le contrôle d'un adulte. Il rassemble des utilisateurs fréquents d'internet et notamment de Youtube et de Skype, mais un adulte doit généralement donner son accord avant l'utilisation de l'ordinateur. Il contrôle également l'inscription sur les réseaux sociaux. Autrement dit, ce premier groupe correspond à une utilisation plutôt ludique d'internet, pour communiquer avec ses camarades, mais contrôlée par les parents.

La seconde classe s'inscrit dans le cadre d'une utilisation plus vertueuse du numérique. Là encore, la présence et l'autorisation d'un adulte sont systématiques, mais les modes d'utilisation concernent avant tout les jeux éducatifs, le travail sur des devoirs, la lecture, ou le travail sur des photos. Ces jeunes n’utilisent en général aucun des réseaux sociaux.

La troisième classe au contraire, concentre avant tout les utilisateurs de réseaux sociaux. Les jeunes disent notamment utiliser Twitter, Instagram, Snapchat, MSN ou Facebook. Ils déclarent également une fréquence d'utilisation élevée de l'ordinateur durant les vacances, mais qui peut aussi concerner le matin avant d'aller à l'école et la pause méridienne. Enfin, ces enfants, qui jouent souvent avec leurs camarades, affirment plus souvent avoir appris seuls à se débrouiller sans l'aide d'un adulte. Ils sont par ailleurs beaucoup moins nombreux à avoir un accord des parents pour utiliser l'ordinateur. Contrairement aux deux précédents, s’esquisse dans ce groupe, une certaine vulnérabilité des jeunes par rapport à internet et à leur pratique du numérique (Jehel, 2015). 


\section{Les usages du numérique par les enseignants}

\section{L'utilisation des TICE par les enseignants}

Globalement, le questionnaire enseignant souligne un intérêt de ces derniers pour les TICE, notamment en ce qui concerne leurs pratiques pédagogiques. La plupart des enseignants (92\%) considèrent les TICE comme une aide à l'enseignement. La fréquence d'utilisation des TICE par les élèves par rapport aux exigences des programmes paraît toutefois insuffisante pour plus de $57 \%$ d'entre eux.

Les enseignants utilisent des ressources technologiques variées dans le cadre de leur fonction. Ainsi, plus des trois quarts mentionnent se servir de divers blogs / forums tenus par des enseignants et du site eduscol. 61\% s’appuient sur le réseau Canopé et $49 \%$ sur la plateforme m@gistère. Environ 65\% des enseignants enseignent des cours d'informatique une fois par semaine alors que $8 \%$ le font plusieurs fois par semaine. A contrario, un enseignant sur cinq n'assure aucun cours d'informatique. En dehors de ce type de cours, un peu plus d'un quart des enseignants utilise les TICE pour enseigner une fois par semaine.

\section{Le matériel mis à disposition pour enseigner}

Une dernière partie du questionnaire visait à recueillir des données concernant le matériel mis à la disposition des enseignants pour enseigner. Or, près d'un quart des enseignants ne disposent d'aucun ordinateur en classe, tandis qu'un peu moins d'un tiers ont une machine et $39 \% \%$ disposent de 2 ordinateurs ou plus. Par ailleurs, près de $70 \%$ des enseignants ont la possibilité d'accéder avec leurs élèves à une salle informatique. L'accès au vidéoprojecteur est le plus usuel (90\% des enseignants). En revanche, ils sont moitié moins à pouvoir utiliser un tableau blanc interactif (TBI) et moins de $10 \%$ à pouvoir utiliser des tablettes.

\section{Quelles compétences numériques des élèves?}

A partir des questions et des problèmes énoncés dans l'enquête, trois domaines dans la maitrise du numérique ont été testés. L'ensemble des questions correspondant au premier concerne des compétences essentiellement techniques d'appropriation de l'outil alors que les questions correspondant au deuxième et au troisième domaine relèvent plus de compétences non techniques.

Les compétences techniques des élèves ont été évaluées par l'intermédiaire de six questions. La première consistait à relier des éléments à l'image correspondante (clavier, enceintes...etc). Ils sont près de $71 \%$ à n'avoir commis aucune erreur. En revanche, à la question de savoir choisir, parmi une liste de propositions, un logiciel de traitement de texte, un peu plus de la moitié seulement $(57,7 \%)$ ont donné une réponse juste. Une troisième question consistait pour les élèves à relier le mot à la bonne image (documentation, matériel numérique...etc), exercice réussi sans erreur pour près de $90 \%$ d'entre eux. De même, les élèves ont eu à indiquer comment «mettre en gras » et « centrer » un texte dans un document word : un tiers des étudiants n'a pas commis d'erreur en répondant, tandis que près d'un élève sur cinq s'est abstenu. Par ailleurs, 63\% des élèves sont parvenus à identifier une adresse URL correcte parmi celles proposées. Enfin, $63 \%$ ont indiqué appeler un adulte lorsqu'ils rencontrent un problème sur l'ordinateur, tandis que 14\% réfléchissent un peu seuls pour le résoudre et 11,2\% arrêtent l'ordinateur. En définitive, afin d'en obtenir une vision plus synthétique, nous avons construit un score de compétences techniques en procédant par 
additition : pour chaque item, nous avons attribué 1 point lorsque la réponse de l'élève était juste et aucun point en cas de réponse fausse. Plus ce score est élevé, plus il signifie que l'élève a de bonnes compétences techniques. Le score moyen s'élève à 4,59, l'écart type à 1,27 .

Deux types de compétences non techniques sont ensuite considérés : celles relatives à la conceptualisation d'internet par les élèves et celles relatives à leur connaissance des droits, ou autrement dit de l'éthique d'internet.

Trois questions portent sur la conceptualisation d'internet. Il a ainsi d'abord été demandé aux élèves ce qu'internet représentait pour eux. Il en ressort que les élèves apprécient particulièrement internet en raison de son côté magique $(32,2 \%)$ et de la possibilité d'avoir accès à plusieurs sites qui donnent des informations sur un même sujet $(28,8 \%)$. Par ailleurs, les élèves ont été questionnés concernant ce qu’ils font pour vérifier qu'une information sur internet est exacte : si 67,2\% des élèves se renseignent auprès d'un adulte et 55,7\% regardent si l'information apparaît sur plusieurs sites internet, plus d'un élève sur cinq (21,4\%) ne vérifie pas et fait confiance à ce qui est écrit. Enfin, près de la moitié des élèves (47\%) ignorent où sont stockées les données issues d'internet, tandis que 27,3\% pensent qu'elles le sont sur leur ordinateur. De même que pour les compétences techniques, un score de compétences non techniques liées à la conceptualisation d'internet a été calculé. Plus il est élevé, plus il indique que les élèves ont de fortes compétences dans ce domaine. Or, ce score a 4,31 pour moyenne, 1,93 pour écart type et 4 pour médiane. Il indique donc que les compétences des élèves en ce qui concerne la conceptualisation d'internet sont relativement faibles.

Quatre questions ont permis d'évaluer les compétences non techniques des élèves en matière d'éthique. Les trois premières, auxquelles plus de $80 \%$ ont indiqué que cela était interdit, concernaient le droit de mettre une photo d'un ami sur internet sans le lui dire, le droit de se moquer d'un camarade par mail et le droit de mettre des renseignements sur les sites de jeu en ligne sans l'accord des parents. La quatrième portait sur l'attitude à adopter lorsque l'élève voit une vidéo de quelqu'un qui se fait frapper : 74,4\% ont répondu qu'il fallait éteindre et en parler à un adulte, 9,6\% qu'il fallait éteindre et faire comme s’ils ne l'avaient pas vue, et 1,4\% qu'il fallait continuer de regarder. Le score de compétences non techniques relatives aux droits sur internet permet d’obtenir une vision plus synthétique des réponses des élèves. La moyenne de ce score s'élève à 3,75, l'écart type à 0,59 et la médiane à 4 . Les élèves ont en définitive de meilleures compétences en ce qui concerne l'éthique que la conceptualisation d'internet.

Une analyse des corrélations entre les trois scores montrent qu'elles sont positives et significativement différentes de 0 , sans être cependant très élevées puisqu'elles sont comprises entre 0,1 et 0,2. Pour identifier les liens entre les caractéristiques des élèves, des enseignants et les compétences utilisées, nous avons cherché à savoir s’il existait des différences significatives de moyenne dans les trois scores de compétences en fonction des informations présentes dans l'enquête. Seules les principales variables significatives sont présentées dans le tableau ci-après. 
Tableau 2 : Analyse des facteurs liés à un score élevé de compétences numériques

\begin{tabular}{|c|c|c|c|}
\hline & \multirow{2}{*}{$\begin{array}{l}\text { Compétences } \\
\text { techniques }\end{array}$} & \multicolumn{2}{|c|}{ Compétences non techniques } \\
\hline & & Conceptualisation & Éthique \\
\hline $\begin{array}{l}\text { Classe : CM2 (versus } \\
\text { CM1) }\end{array}$ & +++ & +++ & +++ \\
\hline \multicolumn{4}{|c|}{ Caractéristique des élèves } \\
\hline Genre : garçon & Ns & Ns & --- \\
\hline Redoublement & --- & Ns & Ns \\
\hline $\begin{array}{l}\text { PCS mère catégorie } \\
\text { favorisée }\end{array}$ & +++ & + & Ns \\
\hline $\begin{array}{l}\text { PCS mère catégorie } \\
\text { moyenne }\end{array}$ & +++ & -- & -- \\
\hline PCS mère défavorisée & -- & Ns & Ns \\
\hline \multicolumn{4}{|c|}{ Profil d'utilisation d'internet en dehors de l'école } \\
\hline Profil élève 1 & Ns & Ns & Ns \\
\hline Profil élève 2 & Ns & Ns & +++ \\
\hline Profil élève3 & - & Ns & --- \\
\hline \multicolumn{4}{|c|}{ Caractéristiques de l'enseignant } \\
\hline Ancienneté 1 à 5 ans & Ns & Ns & --- \\
\hline Ancienneté 6 à 15 ans & Ns & Ns & + \\
\hline Ancienneté 16 à 25 ans & --- & - & Ns \\
\hline Ancienneté > à 25 ans & ++ & Ns & Ns \\
\hline $\begin{array}{l}\text { Enseignant ayant } \\
\text { bénéficié d'une } \\
\text { formation sur les TICE }\end{array}$ & +++ & Ns & Ns \\
\hline $\begin{array}{l}\text { Utilisation fréquente } \\
\text { des TICE dans la classe } \\
\text { (plusieurs fois par } \\
\text { semaine en lien avec } \\
\text { l'informatique) }\end{array}$ & +++ & Ns & Ns \\
\hline \multicolumn{4}{|c|}{ Ressources de l'enseignant } \\
\hline $\begin{array}{l}\text { Au moins trois } \\
\text { ordinateurs dans la salle } \\
\text { de classe }\end{array}$ & +++ & Ns & Ns \\
\hline $\begin{array}{l}\text { Accès une salle } \\
\text { informatique }\end{array}$ & Ns & Ns & Ns \\
\hline TBI & +++ & Ns & Ns \\
\hline Accès direct à internet & +++ & Ns & Ns \\
\hline Tablette & Ns & Ns & Ns \\
\hline
\end{tabular}


Lecture : Un signe positif pour les élèves en CM2 signifie que les moyennes en compétences techniques ou non techniques des élèves de CM2 sont supérieures à celle des élèves de CM1. +++ signifie que les moyennes sont différentes au seuil de $1 \% ;++$ au seuil de $5 \% ;+$ au seuil de $10 \%$ (et réciproquement pour les signes négatifs). Ns indique que la différence n'est pas significative. Les valeurs manquantes sont exclues séparément pour chaque variable.

Le tableau 2 souligne en premier lieu les différences entre compétences techniques et non techniques. Même s’il est délicat d'établir clairement un facteur de causalité, les résultats montrent que les compétences techniques sont assez bien corrélées avec les caractéristiques des élèves et des enseignants. Elles sont plus élevées pour les élèves de CM2, n’ayant pas redoublé et d'origine sociale en général plus favorisée. Du côté des enseignants, ceux qui ont suivi des formations sur les TICE et ceux qui déclarent une utilisation fréquente des TICE ont également des élèves qui ont un niveau plus élevé de compétences techniques. Résultat plus surprenant, les enseignants les plus anciens semblent également ceux qui ont des élèves qui présentent un score de compétences plus élevé. En revanche, le profil d'utilisation à la maison est en général beaucoup moins corrélé avec ces compétences. Seul le troisième profil qui correspondrait à une utilisation plus débridée du numérique et sans contrôle se caractérise par un niveau plus faible de ces compétences. Les ressources numériques de la salle de classe semblent également avoir un effet sur le score de compétences techniques : la présence d'au moins trois ordinateurs dans la classe, l'accès direct à internet ou la présence d'un tableau blanc interactif paraissent être associés à des scores plus élevés pour les élèves.

Les résultats sont beaucoup moins tranchés pour les compétences non techniques, à l'exception du niveau (CM2), qui est dans tous les cas, significatif. Pour les compétences éthiques, les filles ont des scores plus élevés et l'origine sociale semble expliquer un peu plus la conceptualisation d'internet. Les pratiques numériques à la maison paraissent discriminantes pour les compétences éthiques : les élèves qui ont l'utilisation la plus modérée d'internet, la plus contrôlée et en lien avec des motifs scolaires ou culturels (profil 2) sont ceux qui ont les compétences éthiques les plus développées. Inversement, les élèves ayant une utilisation plus débridée à des fins de communication (profil 3) ont le score le plus faible. Les caractéristiques des enseignants se montrent peu discriminantes, si ce n'est pour les compétences éthiques, qui sembleraient moins développées chez les jeunes enseignants.

Pour approfondir l'analyse, des régressions linéaires ont été utilisées pour tester l'effet « toutes choses égales par ailleurs » de toutes les variables. Les résultats, non présentés dans ce travail, confirment d'une part, le rôle de l'origine sociale pour expliquer le score en compétences techniques et d'autre part, l'effet négatif de l'ancienneté de l'enseignant sur le score en compétences éthiques. Les pouvoirs explicatifs des modèles restent cependant relativement faibles, les coefficients de détermination ne dépassant pas $10 \%$. Il est même proche de $1 \%$ pour le score de compétences de conceptualisation d'internet, ce qui souligne la difficulté à en saisir les déterminants. Enfin, il convient de noter, que toutes choses égales par ailleurs, en contrôlant notamment l'origine sociale, l'effet des pratiques numériques à la maison sur les scores de compétences disparaît presque totalement.

\section{Conclusion}

Cette recherche avait pour objectif d'apporter un éclairage sur les pratiques numériques des élèves et le rôle joué par l'institution scolaire dans la construction de leurs compétences 
numériques. Pour cela, une enquête a été menée auprès d'un échantillon de 1400 élèves et d'une cinquantaine d'enseignants. Les données descriptives et les résultats statistiques obtenus permettent de mieux comprendre comment se structure l'acquisition des compétences numériques, même si l'analyse est à approfondir.

Concernant la nature des pratiques numériques des élèves en dehors de l'école, celles-ci apparaissent comme étant variées. De plus, à l'image de ce qu'avait déjà noté Dauphin en 2012, celles-ci sont plutôt de nature ludique, l'utilisation de l'ordinateur à des fins éducatives ne concernant qu'un tiers de l'échantillon. L'enquête montre qu'à l'école, malgré une vision positive du numérique pour l'enseignement, les usages des technologies par les enseignants sont également très variés et dépendent a priori des outils mis à disposition.

La recherche souligne également que les élèves peuvent avoir des conditions d'apprentissage du numérique différentes, notamment dans les trois domaines de compétences que nous avons identifiés. Si dans certains cas, les compétences acquises peuvent d'après nos analyses s'expliquer par les caractéristiques des élèves ou le rôle de l'enseignant, cela n'est pas vrai pour toutes les compétences. Les compétences non techniques et notamment la conceptualisation d’internet, paraissent beaucoup plus complexes à analyser.

Cette recherche demande à être approfondie de manière à stabiliser ces premiers résultats. D’autres techniques statistiques pourraient être mobilisées, telles que des tris croisés ou bien encore des calculs d'attractions entre différentes modalités de réponse. De plus, une collecte sur un nombre plus conséquent de classes nous permettrait de tester un effet " enseignant » par des modélisations multiniveaux (Bressoux, 2007). De même, l'appréhension des compétences numériques des élèves au travers d'un plus grand nombre d'items permettrait d'en réaliser une mesure plus solide. Nous n'avons par ailleurs procédé qu'à une seule prise d'informations, fournissant un instantané à un moment donnée. Or, il serait intéressant de travailler davantage dans une perspective longitudinale afin d'examiner de quelle manière évoluent les compétences numériques des élèves et les facteurs pouvant influer sur ces dernières. Elle pourrait également permettre de mieux appréhender la question de la transférabilité des compétences numériques et de leurs liens avec le niveau scolaire. Cela serait notamment utile pour comprendre la manière dont se construisent les compétences non techniques que nous avons eu des difficultés à expliquer à partir de nos données. Par ailleurs, les conceptions et mesures du numérique à l'école primaire traitées dans cette recherche pourraient être complétées à partir d'un examen approfondi des programmes. La synthèse proposée par Baron et Drot-Delange (2016) souligne en ce sens la pluralité de la pensée informatique développée dans les curricula, qui peut aller jusqu'à l'initiation à la programmation. Il serait en outre pertinent de prolonger ce travail en étudiant plus finement la nature des pratiques numériques des enseignants, par le biais par exemple de l'observation de ces pratiques in situ. Néanmoins, ce travail met en exergue toute l'importance d'approfondir les recherches sur les compétences numériques des élèves, à l'école et en dehors de l'école, afin de mieux saisir les mécanismes à l'œuvre dans le développement de ces compétences par les élèves et leur transférabilité pour faciliter l'apprentissage dans d’autres domaines.

Amélie Duguet, Jean-François Giret, Sophie Morlaix Institut de Recherche sur l’Education (IREDU-ÉA 7318) Université de Bourgogne Franche-Comté (UBFC) 


\section{Bibliographie}

Barette C. (2004). Vers une métasynthèse des impacts des TIC sur l'apprentissage et l'enseignement dans les établissements du réseau collégial québécois, Bulletin Clic, $\mathrm{n}^{\circ} 55$, p. 15-24.

Baron G.-L., Bruillard E. (2008). Technologies de l'information et de la communication et indigènes numériques : quelle situation ? Rubrique de la revue STICEF, vol. 15, p.1-12.

Baron G.-L., Drot-Delange B. (2016). L'informatique comme objet d'enseignement à l'école primaire française ? Mise en perspective historique. Revue française de pédagogie, $\mathrm{n}^{\circ} 195$, p.51-62.

Bernet E., Karsenti T. (2013). Modes d'intégration et usages des TIC au troisième cycle du primaire : une étude multicas. Éducation et francophonie, vol. 41, n¹, p.45-69.

Bressoux P. (2007). L'apport des modèles multiniveaux à la recherche en éducation. Éducation et didactique, vol. 1, n 2, p. 73-88.

Brotcorne P., Valenduc G. (2009). Les compétences numériques et les inégalités dans les usages d'internet. Les cahiers du numérique, vol. 5, n 1, p. 45-68.

Chaptal A. (2008). La réalité des TICE : Un regard critique. In D. Andler, B. Guerry (dir.). Apprendre demain : sciences cognitives et éducation à l'ère numérique. Paris : Hatier, p. 2651.

Collin S., Karsenti T. (2012). Approches théoriques des usages des technologies en éducation : regard critique. Formation et profession, vol. 20, n 3, p. 60-72.

Communauté française de Belgique (1997). Décret définissant les missions prioritaires de l'enseignement fondamental et de l'enseignement secondaire et organisant les structures propres à les atteindre . Moniteur Belge du 24/07/1997 (p. 24 653). Centre de Documentation Administrative de la $\quad$ FWB. $\quad$ [En $\quad$ ligne] http://www.gallilex.cfwb.be/fr/leg_res_01.php?ncda=21557\&referant=102 (consultation 16 avril 2018).

Dadoy M. (1999). Compétence. In A. Akoun, P. Ansart. Dictionnaire de sociologie. Paris : Le Seuil ; Le Robert.

Dauphin F. (2012). Culture et pratiques numériques juvéniles : Quels usages pour quelles compétences ? Questions Vives [en ligne], vol. 7, n 17. https://questionsvives.revues.org/988 (consultation : 8 septembre 2017)

Depover C. (2010). Comprendre et gérer l’innovation. In B. Charlier, F. Henri (dir.). Apprendre avec les technologies. Paris : Presses universitaires de France.

DEPP (2010). Les technologies de l'information et de la communication (TIC) en classe au collège et au lycée : éléments d'usage et enjeux. Les dossiers évaluations et statistiques, $\mathrm{n}^{\circ} 197$. 
Frinking E., Ligtvoet A., Lundin P., Oortwijn W. (2005). The supply and demand of e-skills in Europe. RAND Europe. [En ligne], http://ec.europa.eu/enterprise/ict/policy/doc/eskills-200510-11.rand.pdf (consultation : 15 avril 2018).

Fluckiger C. (2008). L’école à l'épreuve de la culture numérique des élèves. Revue française de pédagogie, $\mathrm{n}^{\circ} 163$, p. 51-61.

Fourgous J.-M. (2010). Réussir l'école numérique. Paris : Ministère de l'éducation nationale.

Granjon F. (2003). Comment résorber la fracture numérique ? Cahiers français, nº 314.

Isnard M., Sautory O. (1994) Les macros SAS d'analyse des données, Document de travail de la Direction des Statistiques Démographiques et Sociales de l'INSEE, $n^{\circ}$ F9405.

Jehel S. (2015). Les pratiques des jeunes sous la pression des industries du numérique. Le Journal des psychologues, $n^{\circ}$ 9, p. 28-33.

Karsenti T., Goyer S., Villeneuve S., Raby C. (2005). L'impact des technologies de l'information et de la communication sur la réussite éducative des garçons à risque de milieux défavorisés. Montréal : CHAIRE de recherche du Canada sur les technologies de l'information et de la communication (TIC) en éducation, CRIFPE.

Michko G. M. (2007). A Meta-analysis of the Effects of Teaching and Learning with Technology on Student Outcomes in undergraduate Engineering Education. Houston : University of Houston.

OCDE (2015). Connectés pour apprendre? Les élèves et les nouvelles technologies, principaux résultats. Paris : OCDE.

Pouts-Lajus S. (2001). « Une question impossible, à propos de l'efficacité des TIC ». [En ligne]: <http://txtnet.com/ote/Une question impossible.htm> (consultation : 16 décembre 2008)

Poyet F. (2009). Impact des TIC dans l'enseignement : une alternative pour l'individualisation ?, Dossier de Veille de l'IFÉ [en ligne], ${ }^{\circ}$ 41, http://ife.ens-lyon.fr/vst/DA-Veille/41-janvier2009.pdf (consultation : 8 septembre 2017)

Prensky M. (2001). Digital Natives, Digital Immigrants. On the Horizon, vol. 9, nº 5, p. 1-6.

Roland N., Choumane M., Vanmeerhaeghe S. (2016). « Les pratiques d'enseignement et d'apprentissage avec le numérique : le cas des tableaux blancs interactifs et des tablettes numériques au sein des écoles de la Ville de Bruxelles ». [En ligne] http://www.gial.be/sites/default/files/actualites/rean_roland2016.pdf $\quad$ (consultation : 8 septembre 2017)

Russel T. (2001). The No Significant Difference Phenomenon: A Comparative Research Annotated Bibliography on Technology for Distance Education ( $5^{\text {th }}$ edition). Chicago : IDECC

Thibert R. (2012). " Pédagogie + numérique = apprentissages 2.0 », Dossier de veille de I'IFÉ [en ligne], n 79, http://ife.ens-lyon.fr/vst/DA-Veille/79-novembre-2012.pdf (consultation : 8 septembre 2017). 
Vandeput E., Henry J. (2012). Pistes pour une mesure de la compétence numérique. Questions Vives, vol.7, n¹7, p.53-70. 
Annexe : Arbre de classification, coordonnées des points sur les axes 1 et 2

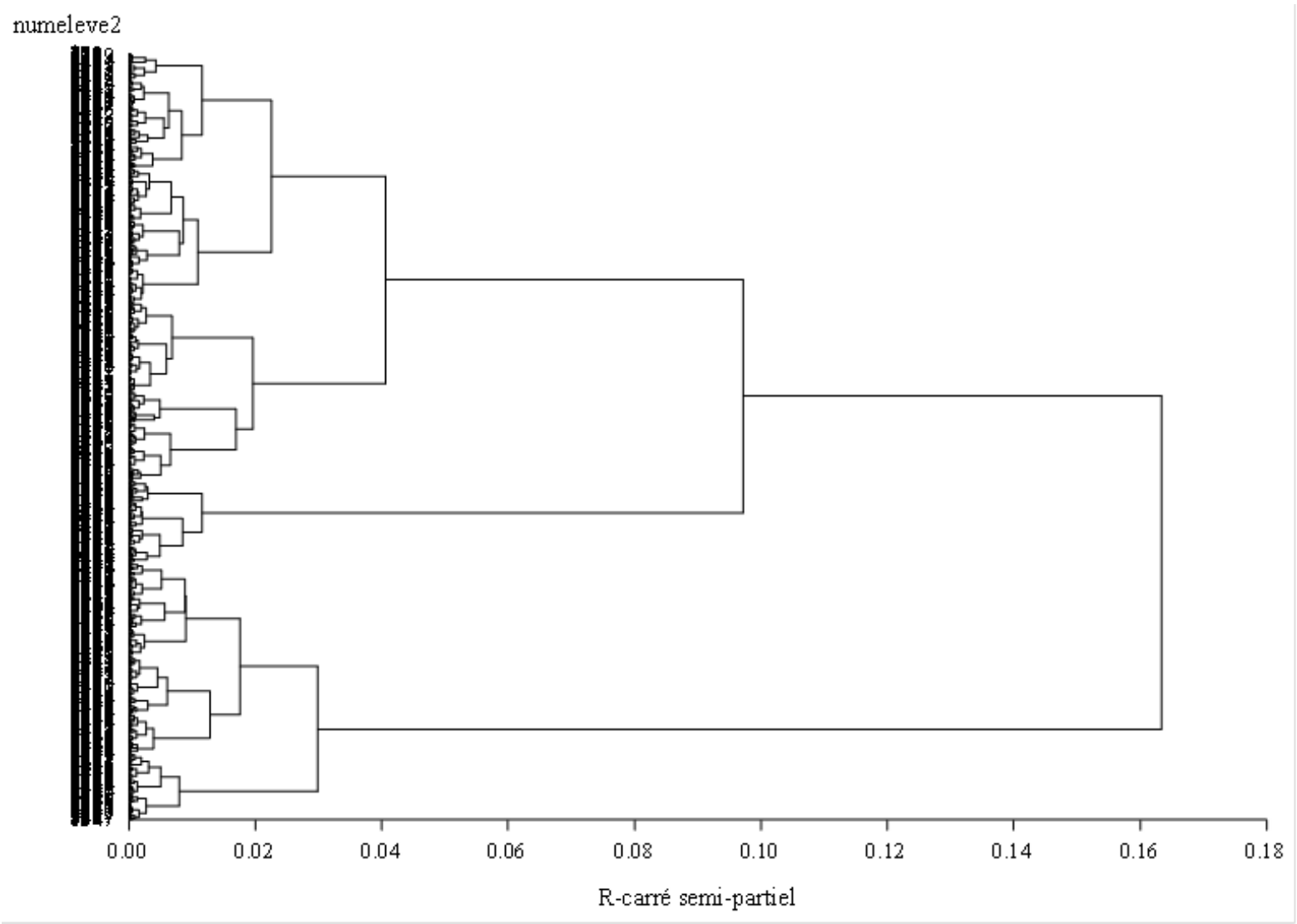

